\title{
USO DE ANÁLISE MULTIVARIADA NA CLASSIFICAÇÃO DAS FONTES HÍDRICAS SUPERFICIAIS DA BACIA HIDROGRÁFICA DO SALITRE ${ }^{1}$
}

\author{
LUIZA T. DE L. BRITO², ADERALDO DE S. SILVA ${ }^{3}$, VAJAPEYAM S. SRINIVASAN ${ }^{4}$, \\ CARLOS DE O. GALVÃO ${ }^{4}$, HANS R. GHEYI ${ }^{5}$
}

\begin{abstract}
RESUMO: Identificar as variáveis de maior significância na qualidade das águas da bacia hidrográfica do Salitre e classificar as fontes hídricas, segundo suas características, foi o objetivo principal deste trabalho. A partir dos resultados obtidos para as barragens/açudes, observa-se que a análise fatorial apresentou três fatores principais que explicam $83,50 \%$ e 74,98\% da variância acumulada, enquanto, para os rios/riachos, explicaram $86,92 \%$ e $71,25 \%$, nos períodos de chuvas e sem chuvas de 2001, respectivamente. As variáveis priorizadas no Fator 1, independentemente das fontes hídricas e dos períodos, estão diretamente relacionadas com a salinidade das águas. As fontes hídricas foram agrupadas em três classes, de forma que a classe $\mathrm{C} 1$ apresentou melhor qualidade das águas. Nos períodos analisados, $61,5 \%$ e $76,9 \%$ das barragens/açudes e $38,5 \%$ e $15,4 \%$ dos rios/riachos foram classificados como $\mathrm{C} 1$, apresentando baixos valores médios para sólidos dissolvidos totais (SDT = 138,$25 ; 209,40$ e 105,$2 ; 129,55 \mathrm{mg} \mathrm{L}^{-1}$ ). Variações nas classes entre as fontes superficiais nos períodos não foram significativas. Esses resultados apontam para a necessidade de medidas de preservação e de conservação dos recursos hídricos dessa bacia hidrográfica.
\end{abstract}

PALAVRAS-CHAVE: impactos ambientais, irrigação, agrupamento.

\section{MULTIVARIATE ANALYSIS USE ON INTERPRETATION OF WATER QUALITY FROM SUPERFICIAL SOURCES OF THE SALITRE RIVER WATERSHED}

\begin{abstract}
This study had the objective of identifying the variables of greatest significance on water quality from the Salitre river watershed and classifying the water sources according to their characteristics. The factorial analysis of the results from dams/streamlets showed three main factors explaining $83.50 \%$ and $74.98 \%$ of the accumulated variance, while from rivers/streamlets, explained $86.92 \%$ and $71.25 \%$ in the raining and drought periods of 2001, respectively. The variables prioritized in the Factor 1, regardless the water sources and the periods are directly related to water salinity. The water sources were grouped into three classes and $\mathrm{C} 1$ showed the best water quality. In the periods studied, $61.5 \%$ and $76.9 \%$ of the dams/streamlets and $38.5 \%$ and $15.4 \%$ of rivers/streamlets were classified as $\mathrm{C} 1$, showing low mean values for total dissolved solids (138.25 and $209.40 \mathrm{mg} \mathrm{L}^{-1} ; 105.2$ and $129.55 \mathrm{mg} \mathrm{L}^{-1}$ ). Variations in the classes among superficial sources in the periods were not significant. These results point out for the needs of measurement of preservation and conservation of water resources of this watershed.
\end{abstract}

KEYWORDS: environmental impacts, irrigation, cluster.

\footnotetext{
${ }^{1}$ Parte da tese de doutorado do primeiro autor.

${ }^{2}$ Enga Agrícola, Pesquisadora da Embrapa Semi-Árido, Fone: (0XX87) 3862.1711, Petrolina - PE, luizatlb@cpatsa.embrapa.br

${ }^{3}$ Eng ${ }^{\mathrm{o}}$ Agrônomo, Doutor, Pesquisador da Embrapa Semi-Árido, Petrolina - PE.

${ }^{4}$ Eng $^{\mathrm{o}}$ Civil, Prof. Dr., Departamento de Engenharia Civil, UFCG, Campina Grande - PB

${ }^{5}$ Eng ${ }^{o}$ Químico, Prof. Dr., Departamento de Engenharia Agrícola, UFCG, Campina Grande - PB

Recebido pelo Conselho Editorial em: 20-9-2004

Aprovado pelo Conselho Editorial em: 21-12-2005
} 


\section{INTRODUÇÃO}

Segundo a Organização Meteorológica Mundial, o consumo mundial de água aumentou mais de seis vezes em menos de um século, mais do que o dobro das taxas de crescimento da população, e continua a crescer com a elevação do consumo dos setores agrícola, industrial e doméstico. Esses estudos demonstram também que, nos próximos anos, a situação global das reservas hídricas tende a piorar, tanto nos aspectos quantitativos quanto nos qualitativos, caso não haja ações enérgicas visando à melhoria da gestão da oferta e da demanda da água para diferentes usos (FREITAS \& SANTOS, 1999).

O Brasil é um dos países de maior disponibilidade hídrica, porém, por conta de suas dimensões geográficas e condições climáticas diferenciadas, algumas regiões sofrem problemas graves de escassez de água, como a região semi-árida do Nordeste e, mais recentemente, grandes metrópoles, como São Paulo, Fortaleza, Recife, entre outras. As soluções desses problemas passam, necessariamente, pela gestão integrada e compartilhada de uso, controle e conservação dos recursos hídricos, que deve ser realizada de forma multidisciplinar e holística, voltada para a conscientização da população na lógica do desenvolvimento sustentado. Para isso, o conhecimento da qualidade das águas torna-se ponto primordial.

No contexto do monitoramento da qualidade das águas, CEBALLOS (1995) aplicou técnicas de análise de componentes principais e de agrupamento às variáveis de qualidade das águas de três açudes, em épocas seca e chuvosa, para avaliar a qualidade sanitária, biológica, física e química das águas, observando que a análise de componentes principais reduziu o número de variáveis analisadas, de 14 para 7, de forma que não alterou as informações sobre a dinâmica interna dos ecossistemas. Esses resultados também permitiram definir medidas de monitoramento desses reservatórios, como a redução de pontos de amostragem de dez para quatro.

SILVA (1997) utilizou análises de componentes principais e de agrupamento para avaliar os impactos ambientais nos solos e nas águas do Projeto de Irrigação Senador Nilo Coelho, em PetrolinaPE, em áreas cultivadas com tomate (Lycopersicun esculentum, M.), utilizando dados de 15 anos de produção, definindo diferentes medidas de redução de impactos em função dos tipos de solos, agricultores, produtividade, uso de fertilizantes e pesticidas.

ORTIZ-ESTARELLES et al. (2001), com auxílio da análise multivariada, avaliaram 25 variáveis de qualidade das águas de uma estação de tratamento, na Espanha, e recomendam que essa metodologia pode ser automatizada e implementada em análise de rotina de laboratórios, utilizando sistema de alerta, visando a prevenir consequiências negativas no uso das águas e/ou minimizar custos de análises de variáveis com baixa participação no conjunto de variáveis que definem a qualidade das águas, aumentando, assim, a eficiência do sistema.

A bacia hidrográfica do Rio Salitre, localizada ao norte da Bahia, nas sub-regiões de Caatinga do Moura (Jacobina), Taquarandi (Mirangaba) e Juazeiro, tem como principal atividade econômica a agricultura irrigada, cultivando principalmente hortifrutícolas para atender aos mercados interno e externo. Nessas áreas, pratica-se uma diversidade de sistemas de produção, a maioria de baixa eficiência de aplicação da água de irrigação e com inadequado manejo dos solos e do uso de fertilizantes e defensivos, favorecendo, conseqüentemente, a poluição das águas; assim, teve-se o objetivo de, neste estudo, avaliar os impactos das atividades agrícolas sobre a qualidade das águas da bacia hidrográfica do Rio Salitre, visando a definir medidas de preservação e conservação dos recursos hídricos, bem como identificar as variáveis de maior significância na qualidade das águas superficiais dessa bacia e classificar as fontes hídricas superficiais de acordo com suas características físicas e químicas, nos períodos de chuvas e sem chuvas, visando a auxiliar na definição de medidas de monitoramento e gestão da qualidade das águas. 


\section{MATERIAL E MÉTODOS}

A bacia hidrográfica do Rio Salitre é uma sub-bacia do Rio São Francisco. Inicia-se na Chapada Diamantina, em Morro do Chapéu - BA, e deságua ao norte, a $20 \mathrm{~km}$ a montante de Juazeiro - BA. Está compreendida entre as latitudes $09^{\circ} 27^{\prime}$ e $17^{\circ} 14^{\prime} \mathrm{S}$ e as longitudes $40^{\circ} 22^{\prime}$ e $41^{\circ} 30^{\prime} \mathrm{W}$, ocupando área de 13.199,7 km², e comprimento do curso principal de $270 \mathrm{~km}$, aproximadamente (CEI, 1986).

\section{Variáveis de qualidade das águas analisadas}

Para a caracterização das águas superficiais da bacia, foram coletadas amostras de água de março e dezembro de 2001, caracterizando os períodos de chuvas (PC) e sem chuvas (PV), respectivamente. As análises foram realizadas em tempo real, utilizando-se de sondas multiparâmetros para as variáveis: temperatura $(\mathrm{T}), \mathrm{pH}$, condutividade elétrica (CE), sólidos dissolvidos totais (SDT), oxigênio dissolvido (OD) e amônio $\left(\mathrm{NH}_{4}{ }^{+}-\mathrm{N}\right)$. Também foram coletadas amostras de águas nessas fontes para a caracterização da composição química, analisando-se as principais concentrações iônicas, como cálcio $\left(\mathrm{Ca}^{++}\right)$, magnésio $\left(\mathrm{Mg}^{++}\right)$, sódio $\left(\mathrm{Na}^{+}\right)$, potássio $\left(\mathrm{K}^{+}\right)$, carbonato $\left(\mathrm{CO}_{3}^{--}\right)$, bicarbonato $\left(\mathrm{HCO}_{3}{ }^{-}\right)$, cloreto $\left(\mathrm{Cl}^{-}\right)$, sulfato $\left(\mathrm{SO}_{4}^{--}\right)$e ferro $\left(\mathrm{Fe}^{++}\right)$, as quais foram realizadas no Laboratório de Solos, Água e Plantas da Embrapa Semi-Árido, em Petrolina - PE. A partir dos dados de algumas variáveis analisadas, foram determinadas a dureza total (DT) e a relação de adsorção de sódio (RAS).

\section{Análise multivariada}

Para a aplicação dos métodos estatísticos, foram construídas matrizes contendo, nas linhas, os diferentes pontos de coleta das águas superficiais e, nas colunas, as respectivas variáveis analisadas nos períodos de chuvas (PC) e sem chuvas (PV) de 2001.

As fontes hídricas foram classificadas utilizando-se de procedimentos de análise multivariada, envolvendo análise fatorial em componentes principais e de agrupamento, em função dos períodos analisados. Para isso, foi utilizado o pacote Statistic Analysis System (SAS), disponibilizado para uso pela Embrapa.

Os fatores ou componentes principais $\left(\mathrm{F}_{\mathrm{i}}\right)$ são independentes, ou seja, ortogonais, determinados a partir de uma transformação linear das variáveis iniciais $X_{i}$, de acordo com o seguinte modelo matemático (ANDRADE, 1989):

$$
\mathrm{X}_{\mathrm{i}}=\mathrm{a}_{\mathrm{i} 1} \mathrm{~F}_{1}+\mathrm{a}_{\mathrm{i} 2} \mathrm{~F}_{2}+\mathrm{a}_{\mathrm{i} 3} \mathrm{~F}_{3}+\ldots+\mathrm{a}_{\mathrm{ik}} \mathrm{F}_{\mathrm{k}}+\mathrm{e}_{\mathrm{i}}
$$

Cada "k" variável observada é descrita linearmente em termos das "k" componentes nãocorrelacionadas $\left(\mathrm{F}_{\mathrm{i}}\right)$; "a $\mathrm{a}_{\mathrm{ik}}$ " são os pesos ou cargas fatoriais da combinação linear, e "e $\mathrm{e}_{\mathrm{i}}$ " corresponde ao erro. As cargas fatoriais " $\mathrm{a}_{\mathrm{ik}}$ " expressam os coeficientes de correlação entre cada uma das variáveis e seu fator, assim, as variáveis mais representativas são aquelas cujas $\mathrm{a}_{\mathrm{ik}}>0,30$; quando a carga fatorial apresenta sinal negativo, significa influência negativa dessa variável no fator (BOUROCHE \& SAPORTA, 1980; ANDRADE, 1989).

A análise de agrupamento consiste em classificar "objetos" em grupos, de acordo com seu grau de similaridade ou de dissimilaridade. Normalmente, para medir o grau de dissimilaridade, utiliza-se da distância euclidiana $\left(d_{a b}\right)$, determinada pela expressão encontrada em BUSSAB et al. (1990), em que $\mathrm{X}_{\mathrm{a}}$ e $\mathrm{X}_{\mathrm{b}}$ são as variáveis, e "p" corresponde ao número de coordenadas envolvidas.

$$
d_{(a, b)}=\left[\sum_{j=1}^{p} \frac{\left(X_{i(a)}-X_{i(b)}\right)^{2}}{p}\right]^{1 / 2}
$$




\section{RESULTADOS E DISCUSSÃO}

As fontes de águas superficiais foram analisadas separadamente de acordo com suas características lênticas e lóticas, isto é, barragens/açudes e os rios/riachos. A partir dos resultados obtidos para as barragens/açudes, observa-se que a análise fatorial apresentou três fatores principais que explicam $83,50 \%$ e 74,98\% da variância total acumulada nos períodos de chuvas e sem chuvas, respectivamente.

O Fator 1 responde por $59,80 \%$ e $52,06 \%$ da variância total e está fortemente representado por variáveis relacionadas com a salinidade das águas ( $\mathrm{Na}, \mathrm{SDT}, \mathrm{Ca}, \mathrm{Cl}, \mathrm{CE}$ ) em ambos os períodos, cujas cargas fatoriais são elevadas $\left(\mathrm{a}_{\mathrm{ik}}>0,800\right)$. O Fator 2 agrupa variáveis como $\mathrm{SO}_{4}, \mathrm{Mg}, \mathrm{DT}, \mathrm{HCO}_{3}, \mathrm{~T}$, no período das chuvas, e $\mathrm{HCO}_{3}$ e T no período sem chuvas, uma vez que o Fe tem influência negativa (Tabela 1). O Fator 3 agrupa, no período das chuvas, as variáveis OD, RAS e Fe, e apenas K no período sem chuvas, uma vez que $\mathrm{OD} \mathrm{e} \mathrm{NH}_{4}{ }^{+}$têm carga negativa. A influência negativa do oxigênio dissolvido (OD) nesse fator pode indicar que, com a redução do volume de água armazenada nas barragens/açudes, devido ao aumento do consumo pelos diversos usos e pelas elevadas taxas evapotranspirométricas, ocorreu maior concentração de solutos, principalmente sais, proporcionando maior decomposição da matéria orgânica, reduzindo as taxas de oxigênio dissolvido nas águas e, ao mesmo tempo, aumentando o íon amônio $\left(\mathrm{NH}_{4}{ }^{+}\right)$, que no período das chuvas está potencializado no Fator 1 (Tabela 1).

Em virtude das elevadas cargas fatoriais das variáveis que participam de cada fator, pode-se denominá-los como: Fator 1 - componente de salinidade; Fator 2 - componente de alcalinidade, e Fator 3 - componente nutricional (BRITO, 2003).

Com relação às fontes hídricas rios/riachos, a análise fatorial em componentes principais apresentou três fatores principais que explicam $86,92 \%$ e $71,25 \%$ da variância total acumulada, nos períodos de chuvas e sem chuvas, respectivamente. No período das chuvas, o Fator 1 agrega variáveis também relacionadas à salinidade das águas, sendo $\mathrm{Cl}\left(\mathrm{a}_{\mathrm{ik}}=0,969\right), \mathrm{Na}\left(\mathrm{a}_{\mathrm{ik}}=0,963\right), \mathrm{SO}_{4}\left(\mathrm{a}_{\mathrm{ik}}=0,897\right)$; no período sem chuvas, as variáveis mais significativas foram DT $\left(\mathrm{a}_{\mathrm{ik}}=0,929\right), \mathrm{HCO}_{3}\left(\mathrm{a}_{\mathrm{ik}}=0,909\right)$ e $\mathrm{Mg}$ $\left(\mathrm{a}_{\mathrm{ik}}=0,838\right)$, embora as demais tenham também expressiva contribuição. O Fator 2 agrega as variáveis $\mathrm{HCO}_{3}, \mathrm{DT}, \mathrm{Mg}, \mathrm{CO}_{3}$ e $\mathrm{Fe}$, com influência negativa, e $\mathrm{Na}, \mathrm{RAS}$ e $\mathrm{Cl}$ nos respectivos períodos, enquanto o Fator 3, em ambos os períodos, contém as variáveis T e OD, tendo OD influência negativa no período das chuvas, e no período sem chuvas essa influência está representada pelo amônio $\left(\mathrm{NH}_{4}\right)$ (Tabela 1).

Pela Tabela 1, observam-se diferenças das variáveis nos Fatores 1 e 2 em relação aos períodos analisados, muito embora, todas essas variáveis estejam diretamente relacionadas com a salinidade das águas, à semelhança das barragens. A maioria das variáveis que compõem o Fator 1 no período das chuvas passa a compor o Fator 2 no período sem chuvas $\left(\mathrm{Cl}, \mathrm{Na}, \mathrm{RAS}\right.$ e $\left.\mathrm{SO}_{4}\right)$. De forma semelhante, as que compõem o Fator 1 no período sem chuvas formam o Fator 2 no período das chuvas $\left(\mathrm{HCO}_{3}, \mathrm{DT}, \mathrm{Mg}, \mathrm{CO}_{3}, \mathrm{Ca}\right.$ e $\left.\mathrm{Fe}\right)$. O Fator 3 é formado pelas variáveis $\mathrm{T}$, $\mathrm{pH}$ e $\mathrm{OD}$, no período das chuvas, sendo que OD tem carga negativa, e por $\mathrm{OD}, \mathrm{T}$ e $\mathrm{NH}_{4}$, no período sem chuvas, com o $\mathrm{NH}_{4}$ com influência negativa, à semelhança dos resultados apresentados para as barragens.

Como os fatores são obtidos de forma hierárquica, medidas prioritárias de gestão e monitoramento de qualidade das águas dessa bacia hidrográfica devem ser implementadas de forma a reduzir a concentração de íons de sais, visando a aumentar a disponibilidade de água na região (BRITO, 2003). 
TABELA 1. Cargas fatoriais, em ordem decrescente, para as variáveis de qualidade das águas das barragens/açudes e dos rios/riachos da bacia hidrográfica do Salitre, nos períodos de chuvas (PC01) e sem chuvas (PV01) de 2001, utilizando análise fatorial.

\begin{tabular}{|c|c|c|c|c|c|c|c|c|c|}
\hline \multicolumn{5}{|c|}{ PC01 } & \multicolumn{5}{|c|}{ PV01 } \\
\hline Variáveis & Fator 1 & Fator 2 & Fator 3 & $\mathrm{CF}^{*}(\%)$ & Variáveis & Fator 1 & Fator 2 & Fator 3 & $\mathrm{CF}^{*}(\%)$ \\
\hline \multicolumn{10}{|c|}{ Barragens/Açudes } \\
\hline $\mathrm{K}$ & 0,954 & 0,049 & $-0,142$ & 93,30 & $\mathrm{Na}$ & 0,885 & 0,264 & 0,201 & 89,39 \\
\hline $\mathrm{NH}_{4}$ & 0,939 & $-0,061$ & $-0,192$ & 92,25 & $\mathrm{SO}_{4}$ & 0,848 & $-0,050$ & $-0,336$ & 83,58 \\
\hline $\mathrm{Na}$ & 0,858 & 0,395 & $-0,271$ & 96,71 & $\mathrm{Cl}$ & 0,845 & 0,467 & 0,050 & 93,47 \\
\hline SDT & 0,831 & 0,452 & $-0,008$ & 89,59 & SDT & 0,837 & 0,491 & 0,123 & 95,87 \\
\hline $\mathrm{Ca}$ & 0,824 & 0,444 & 0,115 & 88,92 & $\mathrm{CE}$ & 0,818 & 0,544 & 0,087 & 97,46 \\
\hline $\mathrm{Cl}$ & 0,811 & 0,481 & $-0,113$ & 90,25 & $\mathrm{Ca}$ & 0,738 & 0,391 & $-0,415$ & 87,13 \\
\hline $\mathrm{CE}$ & 0,789 & 0,576 & $-0,141$ & 97,48 & DT & 0,718 & 0,648 & $-0,118$ & 95,02 \\
\hline $\mathrm{CO}_{3}$ & 0,757 & 0,372 & 0,195 & 75,09 & RAS & 0,668 & $-0,375$ & 0,342 & 70,37 \\
\hline $\mathrm{pH}$ & 0,572 & $-0,026$ & 0,386 & 47,69 & $\mathrm{pH}$ & 0,476 & 0,432 & 0,070 & 41,84 \\
\hline $\mathrm{SO}_{4}$ & 0,065 & 0,896 & $-0,141$ & 82,64 & $\mathrm{CO}_{3}$ & 0,135 & 0,804 & 0,174 & 69,45 \\
\hline $\mathrm{Mg}$ & 0,627 & 0,741 & $-0,043$ & 94,35 & $\mathrm{Mg}$ & 0,656 & 0,697 & 0,003 & 91,68 \\
\hline DT & 0,688 & 0,709 & $-0,014$ & 97,66 & $\mathrm{HCO}_{3}$ & 0,621 & 0,684 & 0,023 & 85,55 \\
\hline $\mathrm{HCO}_{3}$ & 0,634 & 0,656 & $-0,197$ & 87,19 & $\mathrm{~T}$ & 0,099 & 0,434 & 0,025 & 19,93 \\
\hline $\mathrm{T}$ & 0,037 & 0,512 & 0,231 & 31,75 & $\mathrm{Fe}$ & $-0,059$ & $-0,412$ & 0,401 & 33,45 \\
\hline OD & $-0,161$ & $-0,268$ & 0,927 & 95,72 & $\mathrm{~K}$ & 0,288 & 0,421 & 0,676 & 71,76 \\
\hline RAS & 0,528 & $-0,008$ & $-0,684$ & 74,68 & OD & 0,270 & 0,151 & $-0,700$ & 58,63 \\
\hline $\mathrm{Fe}$ & $-0,259$ & $-0,425$ & $-0,776$ & 85,02 & $\mathrm{NH}_{4}$ & 0,270 & 0,151 & $-0,700$ & 58,63 \\
\hline$\vartheta^{* *}(\%)$ & 59,80 & 14,10 & 9,60 & & $\vartheta^{* *}(\%)$ & 52,06 & 14,04 & 8,88 & \\
\hline$\vartheta_{\text {Acum. }}(\%)$ & 59,80 & 73,90 & 83,50 & & $\vartheta_{\text {Acum. }}(\%)$ & 52,06 & 66,09 & 74,98 & \\
\hline \multicolumn{10}{|c|}{ Rios/Riachos } \\
\hline $\mathrm{Cl}$ & 0,969 & 0,139 & $-0,300$ & 96,07 & DT & 0,929 & 0,293 & $-0,083$ & 95,61 \\
\hline $\mathrm{Na}$ & 0,963 & 0,133 & $-0,050$ & 94,80 & $\mathrm{HCO}_{3}$ & 0,909 & 0,116 & $-0,087$ & 84,81 \\
\hline $\mathrm{SO}_{4}$ & 0,897 & 0,216 & $-0,118$ & 86,55 & $\mathrm{Mg}$ & 0,838 & 0,280 & 0,189 & 81,79 \\
\hline SDT & 0,857 & 0,358 & 0,158 & 88,85 & $\mathrm{pH}$ & 0,811 & $-0,087$ & 0,273 & 74,03 \\
\hline $\mathrm{NH}_{4}$ & 0,824 & 0,211 & 0,379 & 86,84 & $\mathrm{CE}$ & 0,789 & $-0,576$ & $-0,088$ & 96,28 \\
\hline $\mathrm{K}$ & 0,802 & 0,415 & 0,235 & 87,08 & K & 0,708 & 0,533 & $-0,120$ & 80,04 \\
\hline $\mathrm{CE}$ & 0,798 & 0,591 & $-0,076$ & 99,21 & SDT & 0,663 & 0,622 & $-0,185$ & 86,18 \\
\hline RAS & 0,674 & $-0,531$ & $-0,399$ & 89,65 & $\mathrm{Ca}$ & 0,658 & $-0,179$ & $-0,528$ & 74,43 \\
\hline $\mathrm{HCO}_{3}$ & 0,216 & 0,904 & 0,086 & 87,17 & $\mathrm{CO}_{3}$ & 0,594 & $-0,117$ & 0,238 & 42,30 \\
\hline DT & 0,493 & 0,852 & 0,136 & 98,88 & $\mathrm{Fe}$ & $-0,741$ & $-0,027$ & 0,003 & 55,08 \\
\hline $\mathrm{Mg}$ & 0,523 & 0,841 & $-0,026$ & 98,27 & $\mathrm{Na}$ & 0,309 & 0,907 & 0,084 & 92,53 \\
\hline $\mathrm{CO}_{3}$ & $-0,065$ & 0,786 & 0,068 & 62,73 & RAS & $-0,219$ & 0,901 & $-0,001$ & 86,06 \\
\hline $\mathrm{Ca}$ & 0,232 & 0,594 & 0,581 & 74,49 & $\mathrm{Cl}$ & 0,470 & 0,813 & $-0,077$ & 88,83 \\
\hline $\mathrm{Fe}$ & $-0,331$ & $-0,837$ & $-0,092$ & 81,87 & $\mathrm{SO}_{4}$ & 0,060 & 0,642 & 0,199 & 45,60 \\
\hline $\mathrm{T}$ & 0,110 & $-0,176$ & 0,939 & 92,56 & OD & $-0,112$ & 0,285 & 0,812 & 75,31 \\
\hline $\mathrm{pH}$ & 0,119 & 0,574 & 0,623 & 73,85 & $\mathrm{~T}$ & 0,093 & 0,063 & 0,168 & 4,13 \\
\hline OD & 0,506 & $-0,275$ & $-0,674$ & 78,64 & $\mathrm{NH}_{4}$ & $-0,044$ & 0,382 & $-0,578$ & 48,17 \\
\hline$\vartheta^{* *}(\%)$ & 53,49 & 23,09 & 10,33 & & $\vartheta^{* *}(\%)$ & 46,13 & 15,89 & 9,23 & \\
\hline$\vartheta_{\text {Ac. }}(\%)$ & 53,49 & 76,59 & 86,92 & & $\vartheta_{\mathrm{Ac}}(\%)$ & 46,13 & 62,02 & 71,25 & \\
\hline
\end{tabular}

${ }^{*} \mathrm{CF}=$ Comunalidade Final; ${ }^{* *} \vartheta=$ Variância explicada por cada Fator. 
A análise de agrupamento classificou as barragens/açudes em três grupos nos períodos analisados, sendo que $61,54 \%$ e $76,92 \%$, correspondendo a 8 e 10 fontes hídricas, respectivamente, foram classificadas como $\mathrm{C} 1$ : águas de melhor qualidade, as quais apresentaram valores médios para sólidos dissolvidos totais relativamente baixos ( $\mathrm{SDT}=138,25$ e 209,40 $\mathrm{mg} \mathrm{L}^{-1}$ ), nos respectivos períodos (Tabela 2), observando-se que praticamente não ocorreram variações nas classes entre períodos. Apenas as barragens TAMB1 e CURT2, classificadas como C2 no período das chuvas, passaram para $\mathrm{C} 1$ no período sem chuvas.

A mudança de classes das barragens/açudes entre os períodos, como conseqüência na melhoria da qualidade das águas, está associada ao carreamento de sais do solo para as fontes hídricas, proporcionando maior concentração de solutos no início do período das chuvas. Como as precipitações pluviométricas ocorridas nessa região foram suficientes para que a água armazenada nesses reservatórios atingisse a cota máxima do sangradouro, ocorreu renovação das águas dessas fontes, melhorando sua qualidade.

Para as fontes classificadas como $\mathrm{C} 2$ e $\mathrm{C} 3$, os valores médios, obtidos para sólidos dissolvidos totais, são elevados (SDT = 772,50; 1.134,50 e de 1.854,0; 2.199,0 mg L $\mathrm{m}^{-1}$ ), independentemente do período (Tabela 2). De acordo com a classificação do BRASIL (1986), no contexto da salinidade, as barragens/açudes agrupados como $\mathrm{C} 1$ podem enquadrar-se na classificação das águas doces (SDT $\leq$ $500 \mathrm{mg} \mathrm{L}^{-1}$ ), sem restrições de uso, e as das classes C2 e C3, como águas salobras (SDT > $500 \mathrm{mg} \mathrm{L}^{-1}$ ) e, portanto, apresentam limitações de uso, embora, muitas vezes, são essas as únicas fontes de água disponíveis nas comunidades.

Embora as variações nas classes definidas pela análise de agrupamento não tenham sido significativas, em função das características próprias e da variabilidade das condições climáticas dessa região, o monitoramento da qualidade das águas pode ser realizado, inicialmente, com freqüência bimestral, em fontes hídricas representativas do ponto de vista das influências antrópicas, como as fontes próximas de áreas irrigadas, de extração mineral, entre outras, como também incluindo variáveis biológicas e, seqüencialmente, definidas as medidas de recuperação e manutenção da qualidade das águas; em relação dos usos preponderantes, pode-se adequar o monitoramento em função do manejo integrado dos fatores do sistema de produção agropecuário utilizado. Entre as medidas para a melhoria da qualidade das águas, podem-se citar aquelas voltadas para a conservação dos solos das áreas circunvizinhas às barragens, reduzindo os processos erosivos e o transporte de elementos à base de nitrogênio e fósforo, que favorecem a eutrofização, além de reduzir a sedimentação dos reservatórios, o que diminuiria a capacidade de armazenamento de água; deve-se evitar, também, que o consumo dos animais seja diretamente na fonte, uma vez que seus dejetos favorecem diferentes processos de poluição.

As fontes rios/riachos também foram classificadas em três classes, de acordo com as características das águas analisadas. Dos 13 pontos amostrados no Rio Salitre e em seus afluentes, apenas dois foram classificados como $\mathrm{C} 1$ e sete como $\mathrm{C} 2$, no período sem chuvas (Tabela 2), cujas águas apresentam melhor qualidade, quando comparadas com as da classe C3. Nos pontos dos rios/riachos TAQUA2, ABREUS e BGALV, ocorreram mudanças de classes, passando de $\mathrm{C} 1$ para $\mathrm{C} 2$, e GOIAB de C2 para C3, caracterizando, assim, degradação da qualidade das águas. Essas variações na qualidade das águas podem estar relacionadas à influência das atividades agrícolas, como a irrigação intensivamente praticada nessas áreas da bacia, sendo também favorecida pela ocorrência da redução da vazão do Rio Salitre no período sem chuvas, proporcionando maior concentração de solutos nas águas. Nesses pontos, os valores médios dos sólidos dissolvidos totais, em ambos os períodos, foram relativamente baixos: variaram de $\mathrm{C} 1=129,55$ a C3 $=664,28 \mathrm{mg} \mathrm{L}^{-1}$ (Tabela 3). 
TABELA 2. Classificação das barragens/açudes e dos rios/riachos da bacia hidrográfica do Salitre nos períodos de chuvas (PC) e sem chuvas (PV) de 2001, utilizando análise de agrupamento.

\begin{tabular}{|c|c|c|c|c|c|}
\hline \multirow[t]{2}{*}{ Município } & \multirow[t]{2}{*}{ Código } & \multicolumn{2}{|c|}{ Localização } & \multicolumn{2}{|c|}{ Classes } \\
\hline & & Latitude & Longitude & $\mathrm{PC}$ & $\mathrm{PV}$ \\
\hline \multicolumn{6}{|c|}{ Barragens/Açudes } \\
\hline Ourolândia & BOURO & $10^{\circ} 58^{\prime} 35^{\prime \prime}$ & $41^{\circ} 05^{\prime} 13^{\prime \prime}$ & $\mathrm{C} 3$ & C3 \\
\hline Morro do Chapéu & TAMB1 & $11^{\circ} 12^{\prime} 51^{\prime \prime}$ & $41^{\circ} 06^{\prime} 09^{\prime}$ & $\mathrm{C} 2$ & $\mathrm{C} 1$ \\
\hline Várzea Nova & CURT2 & $11^{\circ} 17^{\prime} 00^{\prime \prime}$ & $40^{\circ} 54^{\prime} 35^{\prime \prime}$ & $\mathrm{C} 2$ & $\mathrm{C} 1$ \\
\hline Jacobina & BSALINA & $10^{\circ} 55^{\prime} 14^{\prime \prime}$ & $40^{\circ} 47^{\prime} 38^{\prime \prime}$ & $\mathrm{C} 2$ & $\mathrm{C} 2$ \\
\hline Ourolândia & PVERDE & $11^{\circ} 00^{\prime} 09^{\prime}$ & $41^{\circ} 04^{\prime} 33^{\prime}$, & $\mathrm{C} 2$ & $\mathrm{C} 2$ \\
\hline Morro do Chapéu & ROCA1 & $11^{\circ} 20^{\prime} 51^{\prime \prime}$ & $41^{\circ} 02^{\prime} 09^{\prime \prime}$ & $\mathrm{C} 1$ & $\mathrm{C} 1$ \\
\hline Morro do Chapéu & OLHOFB & $10^{\circ} 55^{\prime} 42^{\prime \prime}$ & $41^{\circ} 16^{\prime} 21^{\prime \prime}$ & $\mathrm{C} 1$ & $\mathrm{C} 1$ \\
\hline Várzea Nova & FSACO & $11^{\circ} 11^{\prime} 22^{\prime \prime}$ & $40^{\circ} 47^{\prime} 22^{\prime \prime}$ & $\mathrm{C} 1$ & $\mathrm{C} 1$ \\
\hline Miguel Calmon & TPRACA & $11^{\circ} 14^{\prime} 37^{\prime \prime}$ & $40^{\circ} 43^{\prime} 48^{\prime \prime}$ & $\mathrm{C} 1$ & $\mathrm{C} 1$ \\
\hline Jacobina & OLHOD1 & $10^{\circ} 59^{\prime} 06^{\prime \prime}$ & $40^{\circ} 42^{\prime} 05^{\prime \prime}$ & $\mathrm{C} 1$ & $\mathrm{C} 1$ \\
\hline Jacobina & CANT1 & $11^{\circ} 03^{\prime} 20^{\prime \prime}$ & $40^{\circ} 40^{\prime} 27^{\prime \prime}$ & $\mathrm{C} 1$ & $\mathrm{C} 1$ \\
\hline Jacobina & CANT2 & $11^{\circ} 03^{\prime} 16^{\prime \prime}$ & $40^{\circ} 40^{\prime} 25^{\prime \prime}$ & $\mathrm{C} 1$ & $\mathrm{C} 1$ \\
\hline Mirangaba & TAQUA4 & $10^{\circ} 56^{\prime} 03^{\prime \prime}$ & $40^{\circ} 39^{\prime} 51^{\prime}$, & $\mathrm{C} 1$ & $\mathrm{C} 1$ \\
\hline \multirow{3}{*}{\multicolumn{3}{|c|}{ Número de fontes e freqüência por Classes }} & $\mathrm{C} 1$ & $8(61,5 \%)$ & $10(76,9 \%)$ \\
\hline & & & $\mathrm{C} 2$ & $4(30,8 \%)$ & $2(15,4 \%)$ \\
\hline & & & $\mathrm{C} 3$ & $1(7,7 \%)$ & $1(7,7 \%)$ \\
\hline \multicolumn{6}{|c|}{ Rios/Riachos } \\
\hline Morro do Chapéu & JAC1 & $11^{\circ} 24^{\prime} 39^{\prime \prime}$ & $41^{\circ} 06^{\prime} 49^{\prime \prime}$ & $\mathrm{C} 1$ & $\mathrm{C} 1$ \\
\hline Ourolândia & PINGA & $10^{\circ} 55^{\prime} 45^{\prime}$, & $41^{\circ} 03^{\prime} 59^{\prime \prime}$ & $\mathrm{C} 3$ & $\mathrm{C} 3$ \\
\hline Ourolândia & PINGA1 & $10^{\circ} 55^{\prime} 45^{\prime}$, & $41^{\circ} 03^{\prime} 59^{\prime}$ & $\mathrm{C} 3$ & $\mathrm{C} 3$ \\
\hline Mirangaba & TAQUA1 & $10^{\circ} 53^{\prime} 30^{\prime \prime}$ & $40^{\circ} 41^{\prime} 01^{\prime \prime}$ & $\mathrm{C} 2$ & $\mathrm{C} 2$ \\
\hline Mirangaba & TAQUA2 & $10^{\circ} 53^{\prime} 39^{\prime \prime}$ & $40^{\circ} 41^{\prime} 04^{\prime \prime}$ & $\mathrm{C} 1$ & $\mathrm{C} 2$ \\
\hline Campo Formoso & ABREUS & $10^{\circ} 00^{\prime} 38^{\prime \prime}$ & $40^{\circ} 41^{\prime} 47^{\prime \prime}$ & $\mathrm{C} 1$ & $\mathrm{C} 2$ \\
\hline Campo Formoso & PACUI & $10^{\circ} 04^{\prime} 01^{\prime}$, & $40^{\circ} 43^{\prime} 51^{\prime \prime}$ & $\mathrm{C} 2$ & $\mathrm{C} 2$ \\
\hline Campo Formoso & NASPACU & $10^{\circ} 06^{\prime} 48^{\prime}$ & $40^{\circ} 49^{\prime} 00^{\prime}$ & $\mathrm{C} 2$ & $\mathrm{C} 2$ \\
\hline Campo Formoso & CURRALV & $09^{\circ} 54^{\prime} 54^{\prime \prime}$ & $40^{\circ} 39^{\prime} 07^{\prime \prime}$ & $\mathrm{C} 2$ & $\mathrm{C} 2$ \\
\hline Juazeiro & GOIAB & $09^{\circ} 48^{\prime} 46^{\prime \prime}$ & $40^{\circ} 36^{\prime} 35^{\prime}$, & $\mathrm{C} 2$ & $\mathrm{C} 3$ \\
\hline Juazeiro & ALEGRE & $09^{\circ} 45^{\prime} 14^{\prime \prime}$ & $40^{\circ} 35^{\prime} 51^{\prime \prime}$ & $\mathrm{C} 3$ & $\mathrm{C} 3$ \\
\hline Juazeiro & BGALV1 & $09^{\circ} 29^{\prime} 49^{\prime \prime}$ & $40^{\circ} 39^{\prime} 16^{\prime \prime}$ & $\mathrm{C} 1$ & $\mathrm{C} 2$ \\
\hline Juazeiro & RIOSSF & $09^{\circ} 29^{\prime} 49^{\prime \prime}$ & $40^{\circ} 39^{\prime} 10^{\prime \prime}$ & $\mathrm{C} 1$ & $\mathrm{C} 1$ \\
\hline \multirow{3}{*}{\multicolumn{3}{|c|}{ Número de fontes e freqüência por Classes }} & $\mathrm{C} 1$ & $5(38,5 \%)$ & $2(15,4 \%)$ \\
\hline & & & $\mathrm{C} 2$ & $5(38,5 \%)$ & $7(53,8 \%)$ \\
\hline & & & $\mathrm{C} 3$ & $3(23,0 \%)$ & $4(30,8 \%)$ \\
\hline
\end{tabular}

No contexto da gestão e monitoramento de qualidade das águas, deve-se ressaltar a necessidade de medidas de orientação/capacitação das comunidades, principalmente dos jovens, quanto à preservação dos recursos naturais, em geral, e dos recursos hídricos, em particular, visando à manutenção da capacidade produtiva dos solos e da qualidade das águas. Essas ações, a médio e longo prazos, refletem na saúde do homem e, conseqüentemente, na melhoria da qualidade de vida dessa população e da conservação ambiental. 
TABELA 3. Valores médios das principais características das águas das barragens/açudes e dos rios/riachos da bacia hidrográfica do Salitre nos períodos de chuvas (PC01) e sem chuvas (PV01) de 2001, obtidos pela análise de agrupamento.

\begin{tabular}{|c|c|c|c|c|c|c|c|c|c|c|c|c|c|c|c|}
\hline \multirow{2}{*}{ Classes } & \multirow{2}{*}{ 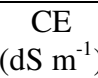 } & \multirow{2}{*}{$\begin{array}{c}\mathrm{SDT} \\
\left(\mathrm{mg} \mathrm{L}^{-1}\right)\end{array}$} & \multirow[t]{2}{*}{$\mathrm{pH}$} & \multirow{2}{*}{$\begin{array}{c}\mathrm{OD} \\
\left(\mathrm{mg} \mathrm{L}^{-1}\right)\end{array}$} & \multirow{2}{*}{\multicolumn{2}{|c|}{$\mathrm{Ca} \quad \mathrm{Mg}$}} & $\mathrm{Na}$ & $\mathrm{K}$ & $\mathrm{HCO}_{3}$ & $\mathrm{SO}_{4}$ & $\mathrm{Cl}$ & \multirow{2}{*}{$\mathrm{Fe}$} & \multirow{2}{*}{$\mathrm{NH}_{4}-\mathrm{N}$} & \multirow{2}{*}{ RAS } & \multirow{2}{*}{$\begin{array}{c}\mathrm{DT} \\
\left.\mathrm{mg} \mathrm{L}^{-1}\right)\end{array}$} \\
\hline & & & & & & & $----(n$ & $\mathrm{ol}_{\mathrm{c}} \mathrm{L}^{-1}$ & & & $-\overline{----}($ & & & & \\
\hline \multicolumn{16}{|c|}{ PC01: Barragens/açudes } \\
\hline 1 & 0,15 & 138,25 & 6,65 & 6,15 & 0,35 & 0,31 & 1,17 & 0,17 & 0,88 & 0,17 & 0,88 & 2,51 & 0,64 & 1,92 & \\
\hline 2 & 0,90 & 772,50 & 8,11 & 7,22 & 1,55 & 4,87 & 3,34 & 0,32 & 3,82 & 0,90 & 4,87 & 0,05 & 1,14 & 1,74 & 321,14 \\
\hline 3 & 2,81 & $1.854,00$ & 7,99 & 5,33 & 2,90 & 12,30 & 11,13 & 1,33 & 5,60 & 0,44 & 21,20 & 0,02 & 3,57 & 4,04 & 759,79 \\
\hline \multicolumn{16}{|c|}{ PV01: Barragens/açudes } \\
\hline 1 & 0,34 & 209,40 & 6,20 & 1,86 & 0,71 & 0,65 & 2,19 & 0,41 & 1,54 & 0,07 & 2,03 & 0,95 & 0,99 & 2,83 & 67,99 \\
\hline 2 & 1,69 & $1.134,50$ & 7,40 & 3,97 & 4,70 & 6,70 & 6,17 & 0,35 & 4,30 & 0,37 & 13,65 & 0,03 & 0,53 & 2,54 & 569,99 \\
\hline 3 & 3,30 & $2.199,00$ & 8,00 & 1,26 & 2,90 & 14,40 & 14,33 & 1,44 & 6,70 & 0,13 & 25,50 & 0,00 & 2,81 & 4,87 & 865,25 \\
\hline \multicolumn{16}{|c|}{ PC01: Rios/riachos } \\
\hline 1 & 0,25 & 105,20 & 6,66 & 6,59 & 0,70 & 1,36 & 0,70 & 0,04 & 1,41 & 0,31 & 0,92 & 1,15 & 0,12 & 0,88 & 102,95 \\
\hline 2 & 0,55 & 394,40 & 7,73 & 4,83 & 1,66 & 3,98 & 0,85 & 0,09 & 4,01 & 0,43 & 1,48 & 0,12 & 0,24 & 0,50 & 281,89 \\
\hline 3 & 0,97 & 650,00 & 7,50 & 8,32 & 1,30 & 5,20 & 2,37 & 0,14 & 3,83 & 1,26 & 4,35 & 0,16 & 0,40 & 1,32 & 324,91 \\
\hline \multicolumn{16}{|c|}{ PV01: Rios/riachos } \\
\hline 1 & 0,19 & 129,55 & 6,33 & 6,95 & 0,58 & 1,18 & 0,61 & 0,04 & 1,06 & 0,30 & 0,91 & 0,89 & 0,20 & 0,80 & 87,75 \\
\hline 2 & 0,57 & 389,70 & 7,78 & 4,99 & 1,50 & 3,82 & 0,67 & 0,10 & 3,80 & 0,27 & 1,51 & 0,07 & 0,19 & 0,41 & 265,96 \\
\hline 3 & 0,99 & 664,28 & 7,71 & 6,67 & 2,30 & 4,35 & 2,24 & 0,17 & 4,12 & 0,66 & 4,46 & 0,06 & 0,33 & 1,23 & 332,80 \\
\hline
\end{tabular}

\section{CONCLUSÕES}

A análise fatorial classificou as variáveis de maior significância na qualidade das águas da bacia hidrográfica do Salitre, priorizando aquelas relacionadas com salinidade; portanto, medidas de gestão e monitoramento de qualidade das águas devem ser implementadas de forma a reduzir a concentração de íons de sais nas águas, aumentando sua disponibilidade na região.

A análise de agrupamento classificou as fontes hídricas de acordo com a qualidade das águas, em três classes. De modo geral, não foram observadas variações significativas nas classes entre os períodos analisados.

Os procedimentos de análise fatorial e de agrupamento poderão facilitar no processo de gestão de recursos hídricos, principalmente relacionado ao monitoramento e de utilização das águas.

\section{REFERÊNCIAS}

ANDRADE, T.A. Métodos estatísticos e econométricos aplicados à análise regional. In: HADDAD, P.R.; FERREIRA, C.M. de C.; BOISIER, S.; ANDRADE, T.A. (Ed.) Economia regional: teorias e métodos de análise. Fortaleza: BNB-ETENE, 1989. p.427-507.

BOUROCHE, J.M.; SAPORTA, G. Análise de dados. Tradução de M. Penchel. Rio de Janeiro: Zahar Editores, 1980. $116 \mathrm{p}$.

BRASIL. Resolução CONAMA nº20, 18 junho de 1986. Brasília: SEMA, 92 p. 1986.

BRITO, L. T. de L. Avaliação de impactos das atividades antrópicas sobre os recursos hídricos da bacia hidrográfica do Rio Salitre - BA, e classificação das fontes hídricas. 2003. 184 f. Tese (Doutorado Recursos Naturais) - Universidade Federal de Campina Grande, Campina Grande, 2003.

BUSSAB, W. de O.; MIAZAKI, E.S.; ANDRADE, D.F. de. Introdução à análise de agrupamentos. In: SIMPÓSIO NACIONAL DE PROBABILIDADE E ESTATÍSTICA, 9., 1990, São Paulo. Anais... São Paulo: ABE, 1990. 106 p. 
CEBALLOS, B.S.O. Utilização de indicadores microbiológicos na tipologia de ecossistemas aquáticos do Trópico Semi-Árido. 1995. 192 f. Tese (Doutorado em Microbiologia Ambiental) Instituto de Ciências Biomédicas, Universidade de São Paulo, São Paulo, 1995.

CENTRO DE ESTATÍSTICA E INFORMAÇÃO (CEI). Avaliação dos recursos hídricos em bacias hidrográficas do Estado da Bahia: Bacia do Rio Salitre. Salvador, 1986. 2v.

FREITAS, M.A.V. de; SANTOS, A.H.M. Importância da água e da informação hidrológica. In: FREITAS, M.A.V. de. (Ed.) O estado das águas no Brasil: perspectivas de gestão e informações de recursos hídricos. Brasília: ANEEL/MME/ MMA-SRH/OMM, 1999. p.13-16.

ORTIZ-ESTARELlES, O.; BIOSCA, Y.M.; HERNANDEZ, M.J.M.; SAGRADO, S.; DOMINGO, E.B. Multivariate data analysis of quality parameters in drinking water. Analyst, Cambridge, v.126, n.1, p.91-6, 2001.

SILVA, A. de S. Impacto ambiental del uso de los recursos suelo y agua en áreas agrícolas bajo riego: estudio aplicado a zonas áridas brasileñas y españolas. 1997. 217 f. Tese (Doutorado em Impacto Ambiental) - Universidad Politécnica de Madrid, Madrid, 1997. 